\title{
A neutron scattering study of strong-symmetric hydrogen bonds in potassium and cesium hydrogen bistrifluoroacetates: Determination of the crystal structures and of the single-well potentials for protons
}

\author{
François Fillaux, ${ }^{1, a)}$ Alain Cousson, ${ }^{2, b)}$ Juan F. R. Archilla, ${ }^{3, \mathrm{c})}$ and John Tomkinson ${ }^{4, \mathrm{~d})}$ \\ ${ }^{1}$ LADIR-CNRS, UMR 7075 Université P. et M. Curie, 2 Rue Henry Dunant, 94320 Thiais, France \\ ${ }^{2}$ Laboratoire Léon Brillouin (CEA-CNRS), C.E. Saclay, 91191 Gif-sur-Yvette Cedex, France \\ ${ }^{3}$ Group of Nonlinear Physics, Departamento de Física Aplicada I, ETSI Informática. Avda \\ Reina Mercedes s/n, 41012 Sevilla, Spain \\ ${ }^{4}$ Rutherford Appleton Laboratory, Chilton, Didcot OX11 OQX, United Kingdom
}

(Received 22 February 2008; accepted 21 April 2008; published online 23 May 2008)

\begin{abstract}
The crystal structures of potassium and cesium bistrifluoroacetates, $\mathrm{KH}\left(\mathrm{CF}_{3} \mathrm{COO}\right)_{2}$ and $\mathrm{CsH}\left(\mathrm{CF}_{3} \mathrm{COO}\right)_{2}$, respectively, were determined at room and cryogenic temperatures with the single crystal neutron diffraction technique. The crystals belong to the monoclinic space groups, $I 2 / a$ and $A 2 / a$, respectively, and there is no evidence of any structural phase transition. In both crystals, trifluoroacetate entities in centrosymmetric dimers are linked by very short hydrogen bonds lying across a center of inversion. The thermal parameters provide no evidence of any double minimum potential for hydrogen bond protons. Single-minimum potentials were determined via best fitting to the inelastic neutron scattering spectral profiles of the stretching vibrations. They comprise a narrow well for the ground state and a very broad quasiharmonic well for excited states. The spread out of the wave functions of these states shows that protons are no longer confined between the oxygens. Presumably, they are attracted by the lone pairs of oxygen atoms. These potentials emphasize the covalent nature of the $\mathrm{OO}$ bond and the ionic character of the hydrogen bond proton.
\end{abstract}

(c) 2008 American Institute of Physics. [DOI: 10.1063/1.2927353]

\section{INTRODUCTION}

Hydrogen bonds are ubiquitous in nature, so the concept of hydrogen bonding is of fundamental importance in many disciplines across physics, chemistry, and biology. However, there are many unresolved problems about the quantum nature of this particular chemical bond. ${ }^{1-5}$ The enormous range of energy, from about 2 to $40 \mathrm{kcal} \mathrm{mol}^{-1}$, may be part of the difficulty, for hydrogen bonds might not be amenable to a single concept. In particular, current controversies are focused on very strong hydrogen bonds (VSHBs) OHO whose lengths are shorter than $\approx 2.45 \AA \AA^{6-10}$ These bonds exhibit exceptional physical and chemical properties. In addition, the possible role of VSHBs in enzymatic catalysis broadens their potential significance. ${ }^{11-18}$ The purpose of this present paper is to enlighten on some structural and dynamical features of these bonds.

It is a widespread opinion that structural, $^{7-10}$ dynamical, ${ }^{19}$ and magnetic ${ }^{16,20-22}$ properties of VSHBs in crystals are inconsistent with the relatively long-range attraction, predominantly electrostatic in nature, attributed to weak hydrogen bonds. Gilli and co-workers, ${ }^{8-10}$ or Tian and $\mathrm{Li}^{23}$

\footnotetext{
a) Author to whom correspondence should be addressed. URL: http:// ulysse.glvt-cnrs.fr/ladir/pagefillaux.htm. Electronic mail: fillaux@glvtcnrs.fr.

${ }^{b}$ Electronic mail: cousson@llb.saclay.cea.fr.

${ }^{c)}$ Electronic mail: archilla@us.es.

${ }^{d)}$ Electronic mail: j.tomkinson@rl.ac.uk.
}

have suggested a resonance between covalent structures (e.g., $\mathrm{O}-\mathrm{H} \cdots \mathrm{O} \leftrightarrow \mathrm{O} \cdots \mathrm{H}-\mathrm{O}$ ), but Sørensen et $a .^{24}$ have opposed a counterexample to this view.

From the dynamical viewpoint, one can distinguish "single-well hydrogen bonds" (SWHBs) and "low barrier hydrogen bonds" (LBHBs), ${ }^{12-14,21,22}$ depending on the shape of the potential for protons moving along the $\mathrm{O} \cdots \mathrm{O}$ direction. (In the present paper, this coordinate is $x_{a}$ and the eigenstate vector $\left|x_{a}\right\rangle_{n}$ is at $h \nu_{a n}$.) These potentials could explain different chemical reactivities, for example, isotopic fractionation factors, ${ }^{21}$ but experimental or theoretical evidences are rather scarce.

The prototypical intramolecular strong-symmetric hydrogen bond (SSHB) in the crystal of potassium hydrogen maleate, $\mathrm{KH}(\mathrm{OOC}-\mathrm{CH}=\mathrm{CH}-\mathrm{COO})$ or $\mathrm{KHM}$, has been thoroughly investigated with $\mathrm{X}$-ray or neutron diffraction, ${ }^{25-27}$ infrared and Raman, ${ }^{28-31}$ inelastic neutron scattering (INS) ${ }^{27,32-34} \mathrm{NMR},{ }^{35,36}$ and calorimetry. ${ }^{37}$ The linear hydrogen bond is very short $\left[R_{\mathrm{OO}}=2.427(1) \AA\right.$ at $\left.5 \mathrm{~K}\right]$ and crystallographically symmetrical. The probability density of the hydrogen bond proton located at the center, visualized as a thermal ellipsoid, accords with a single well. INS spectra of single crystals of various H/D derivatives have revealed a large number of $\left|x_{a}\right\rangle_{n}$ states (at least 7 between 500 and $1300 \mathrm{~cm}^{-1}$ ) consistent with a symmetric funnelshaped potential (Fig. 1). To the best of our knowledge, this potential has never been confronted with theoretical quantum chemistry, so the interpretation is circumstantial. Wilson et al. ${ }^{38}$ by using plane-wave density functional theory, cal- 

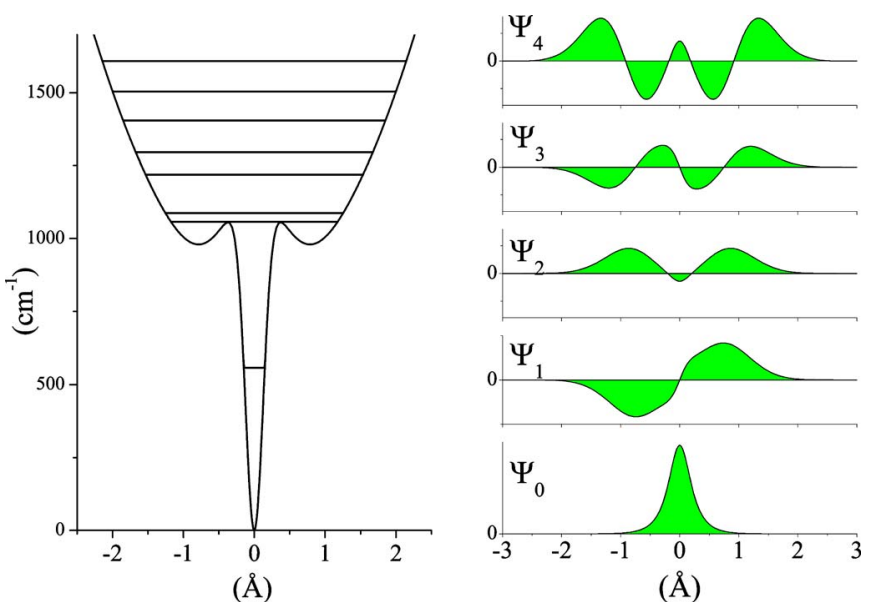

FIG. 1. (Color online) Potential function, eigenstates, and wave functions along the stretching coordinate of the hydrogen bond proton in potassium hydrogen maleate, after Ref. 27.

culated the potential energy in this crystal, but only for a very limited range of proton displacements $\left(\Delta x_{a} \approx \pm 0.1 \AA\right)$ that cannot make any contact with the funnel shape. ${ }^{39}$ On the other hand, calculations of the isolated maleate ion lead to a LBHB at variance with the crystal structure. ${ }^{38,40,41}$ We are not aware of any theoretical modeling of very large proton displacements $(\sim 2 \AA)$ for KHM in the crystal field.

To the best of our knowledge, the funnel potential of KHM is unique. Could it be of general relevance to SSHBs? That is the question at issue in this present paper. This question is controversial since a new intramolecular VSHB $\left[R_{\mathrm{OO}}=2.388(5) \AA\right.$ at $\left.20 \mathrm{~K}\right]$ has been reported in the crystal of 4-cyano-2,2,6,6-tetramethyl-3,5-heptanedione (4CTH). ${ }^{22}$ In contrast to KHM, the hydrogen bond is not linear $\left(r_{\mathrm{OH}} \approx 1.22 \AA, \mathrm{OHO} \approx 157^{\circ}\right)$ and, needless to say, noncentrosymmetric. An INS band at $371 \mathrm{~cm}^{-1}$, tentatively assigned to $\left|x_{a}\right\rangle_{1}$, along with solid-state NMR measurements, were regarded as possible evidences of a LBHB. However, there are solid counterarguments to this conclusion. (i) These authors point out that "the neutron [diffraction] data better fit a single anisotropic thermal ellipsoid...," so a single well is more plausible, even though the academic case of a potential barrier below the zero-point energy cannot be excluded. (ii) The same authors report that $a b$ initio calculations of the isolated molecule lead to an imaginary frequency suggesting a potential barrier at the center. However, such calculations are not conclusive. For example, they lead to a LBHB for the isolated maleate ring that is at variance with the SWHB observed in the crystal. One cannot exclude the important role of the crystal field in the calculation of the optimized geometry. In addition, spectroscopic observables are representative of an effective potential that can be different from the BornOppenheimer potential. (iii) The INS spectra were measured only below $640 \mathrm{~cm}^{-1}$, so it is unknown whether higher transitions corroborate a double well. (iv) Even for the methyl deuterated derivative, the neutron flux scattered by all atoms is much greater than that scattered by the single hydrogen bond proton, so the contrast of intensities is not sufficient to establish an assignment scheme. (v) The invaluable information provided by infrared and Raman is missing. These nu- merous drawbacks show that the band assignment and the double well proposed by these authors deserve reservations. Furthermore, it is widely accepted that the potential barrier and the hydrogen bond length should decrease simultaneously. A double well for a hydrogen bond shorter than that in KHM would be, therefore, rather odd, unless it would be due to the nonlinear OHO geometry. Unfortunately, the large number of tetramethyl groups is a serious hurdle for further INS studies of this interesting system.

In the present paper, in order to fully exploit the contrast of intensities for infrared, Raman, and INS, we consider crystals in which hydrogen bond protons are singled out. Potassium, or cesium, hydrogen bistrifluoroacetate, $\mathrm{KH}\left(\mathrm{CF}_{3} \mathrm{COO}\right)_{2}[(\mathrm{KTFA})]$ or $\mathrm{CsH}\left(\mathrm{CF}_{3} \mathrm{COO}\right)_{2}[(\mathrm{CTFA})]$, are known examples of "intermolecular" SSHBs. ${ }^{42}$ At room temperature, trifluoroacetate dimers, $\mathrm{H}\left(\mathrm{CF}_{3} \mathrm{COO}\right)_{2}^{-}$, are linked by crystallographically symmetric hydrogen bonds with $R_{\mathrm{OO}}=2.435(7)$ or $2.38(3) \AA$, respectively. The vibrational spectra of these salts at a very low temperature ${ }^{19,43,44}$ evidence several $\left|x_{a}\right\rangle_{n}$ states in the same frequency range as for KHM (500-1100 $\left.\mathrm{cm}^{-1}\right)$ but with quite different profiles of intensity. In a previous work, ${ }^{44}$ it was suggested that CTFA could be a LBHB case, but there is no neutron diffraction data to support this proposal. We report below single crystal neutron diffraction measurements consistent with single wells for protons at any temperature. This result prompts us to revisit the vibrational spectra, and we propose a funnel potential largely inspired by that shown in Fig. 1 but not quite the same. We tentatively rationalize these various potentials with bare protons $\mathrm{H}^{\oplus}$ weakly bound to ${ }^{\ominus} \mathrm{O}-\mathrm{O}^{\ominus}$ bonds.

This paper is organized as follows. The crystal structures at room and cryogenic temperatures are presented in Sec. II. In Sec. III, we propose an assignment scheme for the $\left|x_{a}\right\rangle_{n}$ states based on infrared, Raman, and INS spectra. In Sec. IV, we elaborate on the theoretical framework for proton dynamics, we determine the best potential, and we examine some consequences to the quantum nature of SSHBs.

\section{CRYSTAL STRUCTURES}

Single crystals were obtained by slow crystallization from aqueous solutions. Approximately cubic samples $\left(3 \times 3 \times 3 \mathrm{~mm}^{3}\right)$ were loaded into aluminum containers and then mounted in a cryostat. Measurements (see Table I) were carried out with the Stoe four-circle diffractometer 5C2 at the Orphée reactor (Laboratoire Léon-Brillouin). ${ }^{45}$ Data analysis was carried out with the computer package CRYSTALS. ${ }^{46,47}$ Absorption corrections were ignored.

The structures at room temperature are similar to those previously determined with $\mathrm{x}$-rays. ${ }^{42}$ At low temperatures, the lattice parameters (Table I) and the positional parameters (Tables II and III) are not significantly changed. There is no evidence of any phase transition or proton disorder. The potassium and cesium salts belong to the $I 2 / a$ and $A 2 / a$ monoclinic space groups, respectively, both with four dimer entities in the unit cell. The crystallographically equivalent TFAs of a dimer are linked by short linear hydrogen bonds 
TABLE I. Neutron single crystal diffraction data and structure refinement for potassium and cesium hydrogen bistrifluoroacetates. $\lambda=0.8305 \AA$. Space groups monoclinic $I 2 / a$ for potassium and $A 2 / a$ for cesium. Both with $Z=4$. The criterion for used reflections was $I>3 \sigma(I)$. The variance for the last digit is given in parentheses. In all cases, 76 parameters were used in refinement on $\mathrm{F}$.

\begin{tabular}{|c|c|c|c|c|}
\hline & \multicolumn{2}{|c|}{$\mathrm{KH}\left(\mathrm{CF}_{3} \mathrm{COO}\right)_{2}$} & \multicolumn{2}{|c|}{$\mathrm{CsH}\left(\mathrm{CF}_{3} \mathrm{COO}\right)_{2}$} \\
\hline & $20 \mathrm{~K}$ & $298 \mathrm{~K}$ & $14 \mathrm{~K}$ & $298 \mathrm{~K}$ \\
\hline$a(\AA)$ & $8.68(1)$ & $8.78(1)$ & $13.44(1)$ & $13.623(8)$ \\
\hline$b(\AA)$ & $10.023(9)$ & $10.18(1)$ & $4.942(9)$ & $5.033(3)$ \\
\hline$c(\AA)$ & $9.146(9)$ & $9.28(1)$ & $14.35(1)$ & $14.741(6)$ \\
\hline$\beta\left({ }^{\circ}\right)$ & $100.36(8)$ & 99.96(9) & $112.88(9)$ & $112.46(9)$ \\
\hline$V\left(\AA^{3}\right)$ & 782.6 & 817.0 & 878.4 & 934.0 \\
\hline$D_{x}\left(\mathrm{Mg} \mathrm{m}^{-3}\right)$ & 2.259 & 2.164 & 2.722 & 2.559 \\
\hline Measured reflections & 1979 & 2164 & 3476 & 3897 \\
\hline Independent reflections & 1769 & 1472 & 1977 & 2096 \\
\hline Used reflections & 1503 & 1142 & 1557 & 1062 \\
\hline$R_{\text {int }}$ & 0.037 & 0.038 & 0.048 & 0.064 \\
\hline$R$ factor & 0.040 & 0.045 & 0.0399 & 0.043 \\
\hline Weighted $R$ factor & 0.042 & 0.029 & 0.0345 & 0.040 \\
\hline Goodness of fit & 1.070 & 1.088 & 1.049 & 1.079 \\
\hline Extinction coefficient & $22.7(7)$ & $17.2(8)$ & $10.0(3)$ & $11.2(9)$ \\
\hline
\end{tabular}

lying across a center of inversion (Figs. 2 and 3). The conformations of the TFA entities are quite different for the two salts: A C-F bond is trans to the $\mathrm{C} 2 \mathrm{O} 2$ bond for KTFA, while a $\mathrm{C}-\mathrm{F}$ bond is perpendicular to the carboxylic plane for CTFA. At room temperature, all temperature factors increase substantially (see Table IV, Fig. 2, Table V, and Fig. 3). They are 10-15 times greater for heavy atoms

TABLE II. Atomic positions and isotropic temperature factors for $\mathrm{KH}\left(\mathrm{CF}_{3} \mathrm{COO}\right)_{2}$ at $20 \mathrm{~K}$ (first lines) and $298 \mathrm{~K}$ (second lines). The variance for the last digit is given in parentheses.

\begin{tabular}{|c|c|c|c|c|}
\hline Atom & $x / a$ & $y / b$ & $z / c$ & $U$ (iso) $\left(\AA^{2}\right)$ \\
\hline \multirow[t]{2}{*}{$\mathrm{K}(1)$} & 0.2500 & $0.47035(13)$ & 1.0000 & 0.0022 \\
\hline & 0.2500 & $0.4691(2)$ & 1.0000 & 0.0306 \\
\hline \multirow[t]{2}{*}{$C(1)$} & $0.08108(6)$ & $0.65992(5)$ & $0.61071(5)$ & 0.0020 \\
\hline & $0.0771(1)$ & $0.66238(9)$ & $0.61201(9)$ & 0.0306 \\
\hline \multirow[t]{2}{*}{$C(2)$} & $-0.04133(6)$ & $0.66059(5)$ & $0.71503(6)$ & 0.0020 \\
\hline & $-0.04356(9)$ & $0.66241(8)$ & $0.71512(8)$ & 0.0260 \\
\hline \multirow[t]{2}{*}{$\mathrm{O}(1)$} & $-0.02359(7)$ & $0.58604(6)$ & $0.82262(7)$ & 0.0040 \\
\hline & $-0.02706(13)$ & $0.58871(11)$ & $0.81966(11)$ & 0.0333 \\
\hline \multirow[t]{2}{*}{$\mathrm{O}(2)$} & $-0.15042(7)$ & $0.74496(6)$ & $0.67180(7)$ & 0.0045 \\
\hline & $-0.15011(14)$ & $0.74556(14)$ & $0.67428(14)$ & 0.0426 \\
\hline \multirow[t]{2}{*}{$\mathrm{F}(1)$} & $0.15118(7)$ & $0.78039(7)$ & $0.61351(7)$ & 0.0050 \\
\hline & $0.14415(18)$ & $0.78021(15)$ & $0.61321(18)$ & 0.0519 \\
\hline \multirow[t]{2}{*}{$\mathrm{F}(2)$} & $0.19203(7)$ & $0.56895(7)$ & $0.65128(7)$ & 0.0046 \\
\hline & $0.18745(15)$ & $0.57426(17)$ & $0.65321(17)$ & 0.0490 \\
\hline \multirow[t]{2}{*}{$\mathrm{F}(3)$} & $0.01440(8)$ & $0.63619(7)$ & $0.47010(7)$ & 0.0046 \\
\hline & $0.01325(19)$ & $0.63720(16)$ & $0.47512(13)$ & 0.0474 \\
\hline \multirow[t]{2}{*}{$\mathrm{H}(1)$} & -0.2500 & 0.7500 & 0.7500 & 0.0166 \\
\hline & -0.2500 & 0.7500 & 0.7500 & 0.0574 \\
\hline
\end{tabular}

TABLE III. Atomic positions and isotropic temperature factors for $\mathrm{CsH}\left(\mathrm{CF}_{3} \mathrm{COO}\right)_{2}$ at $14 \mathrm{~K}$ (first lines) and $298 \mathrm{~K}$ (second lines). The variance for the last digit is given in parentheses.

\begin{tabular}{llllc}
\hline \hline Atom & \multicolumn{1}{c}{$x / a$} & \multicolumn{1}{c}{$y / b$} & \multicolumn{1}{c}{$z / c$} & $U($ iso $)\left(\AA^{2}\right)$ \\
\hline $\mathrm{Cs}(1)$ & -0.2500 & $0.21541(18)$ & 0.0000 & 0.0013 \\
& -0.2500 & $0.2170(4)$ & 0.0000 & 0.0377 \\
$\mathrm{~F}(1)$ & $-0.01883(5)$ & $0.61975(15)$ & $0.15253(5)$ & 0.0059 \\
& $-0.01376(14)$ & $0.5971(4)$ & $0.15586(14)$ & 0.0652 \\
$\mathrm{~F}(2)$ & $0.10983(5)$ & $0.39738(15)$ & $0.26553(5)$ & 0.0069 \\
& $0.11435(18)$ & $0.3806(5)$ & $0.26110(12)$ & 0.0761 \\
$\mathrm{~F}(3)$ & $0.14684(5)$ & $0.72718(14)$ & $0.18559(5)$ & 0.0063 \\
& $0.14651(17)$ & $0.7048(4)$ & $0.18308(15)$ & 0.0722 \\
$\mathrm{O}(1)$ & $0.16871(5)$ & $0.28677(13)$ & $0.08444(5)$ & 0.0050 \\
& $0.16517(9)$ & $0.2837(3)$ & $0.0796(1)$ & 0.0445 \\
$\mathrm{O}(2)$ & $-0.00501(5)$ & $0.18925(14)$ & $0.05235(5)$ & 0.0045 \\
& $-0.00336(8)$ & $0.1803(3)$ & $0.0543(1)$ & 0.0442 \\
$\mathrm{C}(1)$ & $0.07995(4)$ & $0.52050(11)$ & $0.17554(4)$ & 0.0031 \\
& $0.08238(8)$ & $0.5005(2)$ & $0.17453(7)$ & 0.0401 \\
$\mathrm{C}(2)$ & $0.08431(4)$ & $0.31424(11)$ & $0.09597(4)$ & 0.0027 \\
& $0.08415(6)$ & $0.30433(18)$ & $0.09498(7)$ & 0.0312 \\
$\mathrm{H}(1)$ & 0.0000 & 0.0000 & 0.0000 & 0.0166 \\
& 0.0000 & 0.0000 & 0.0000 & 0.0592 \\
\hline \hline
\end{tabular}

and only three to five times greater for protons. For heavy atoms, the ellipsoids suggest librations around the $\mathrm{C}-\mathrm{C}$ bonds.

Hydrogen bond lengths are identical at low temperature, $R_{\mathrm{OO}}=2.436(3) \AA$ for KTFA or 2.436(4) $\AA$ for CTFA, and very close to $R_{\mathrm{OO}}=2.427(1) \AA$ for KHM. Differences of about twice the variance are not significant. These SSHBs are, therefore, unaffected by the crystal stacking, the TFA conformation, or resonance within the maleate ring. At room temperature, $R_{\mathrm{OO}}=2.432(3) \AA$ for KTFA and 2.444(2) $\AA$ for CTFA. These temperature effects are not significant, com-
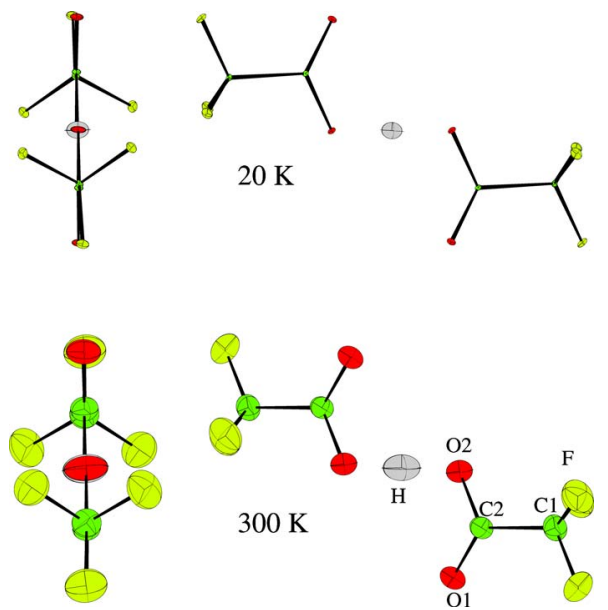

FIG. 2. (Color online) Comparison of the hydrogen bistrifluoroacetate entities of the potassium salt at 20 and $300 \mathrm{~K}$. Right: Projection onto the mean plane of the carboxylic entities. Left: View along the hydrogen bond direction. The ellipsoids correspond to $50 \%$ probabilities for atoms. 

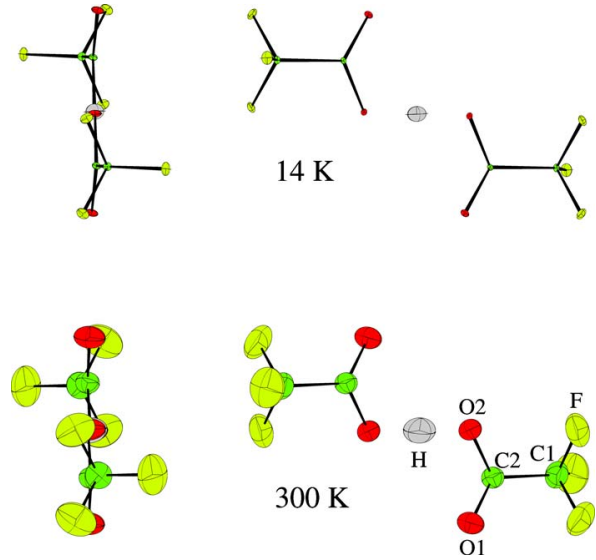

FIG. 3. (Color online) Comparison of the hydrogen bistrifluoroacetate entities of the cesium salt at 14 and $300 \mathrm{~K}$. Right: Projection onto the mean plane of the carboxylic entities. Left: View along the hydrogen bond direction. The ellipsoids correspond to $50 \%$ probabilities for atoms.

pared to the estimated variances. The difference between the two salts is smaller than that previously determined with $\mathrm{x}_{\text {-rays. }}{ }^{42}$ This is likely a consequence of the huge $\mathrm{X}$-ray cross section of $\mathrm{Cs}$ atoms.

There is no evidence of any splitting of the proton sites that could suggest a double well, and the thermal factors at low temperatures are consistent with a quasiharmonic single well for the ground state. Supposing an oscillator mass of 1 amu (see below Sec. IV), the mean-square amplitudes for the three proton modes at frequencies of about $800 \mathrm{~cm}^{-1}$ $\left(\nu_{a}\right), 1250 \mathrm{~cm}^{-1}$ (out-of-plane bending, $\gamma$ ), and $1600 \mathrm{~cm}^{-1}$ (in-plane bending, $\delta$ ), ${ }^{44}$ are $u_{0 \nu}^{2} \approx 0.021 \AA^{2}, u_{0 \gamma}^{2} \approx 0.013 \AA^{2}$, and $u_{0 \delta}^{2} \approx 0.010 \AA^{2}$. The averaged value $\left\langle u_{0}^{2}\right\rangle \approx 0.015 \AA^{2}$ compares to the thermal factors $U($ iso $)=0.0166 \AA^{2}$ in Tables II and III, or, equivalently, to the averaged anisotropic factors $U=\left(U_{11}+U_{22}+U_{33}\right) / 3 \approx 0.017 \AA^{2}$ in Tables IV and $\mathrm{V}$. The difference $U-\left\langle u_{0}^{2}\right\rangle \approx 0.002 \AA^{2}$ is similar to the thermal parameters for oxygen atoms. There is no evidence of any double well.

It is noticeable that the thermal factors for protons at room temperature are very close to the sum of the thermal factors determined at low temperature and those for $\mathrm{O}$ atoms at room temperature. Figures 2 and 3 show that the thermal ellipsoids for $\mathrm{O}$ atoms are very small at low temperatures, compared to those for protons, while at room temperature, the thermal ellipsoids for protons and oxygens are similar. This suggests that the mean-square amplitudes for protons are largely temperature independent and temperature effects primarily arise from convolution with the probability density of $\mathrm{O}$ atoms. This is consistent with rather deep wells for protons, such that the populations of excited states remain to be negligible at room temperature. There is, therefore, no evidence of any double well, even at room temperature. On the other hand, the thermal population of phonons at low frequencies should account for the increase in the thermal factors in heavy atoms at room temperature.

\section{INS BAND PROFILES}

INS band intensities are proportional to the nuclear cross sections for incoherent scattering, on the one hand, and to the

TABLE IV. Thermal parameters in $\AA^{2}$ units for $\mathrm{KH}\left(\mathrm{CF}_{3} \mathrm{COO}\right)_{2}$ at $20 \mathrm{~K}$ (first lines) and $298 \mathrm{~K}$ (second lines). The variance for the last digit is given in parentheses. The thermal parameters account for the variation of the contribution of each atom to Bragg's peak intensities through the thermal factor $T^{\text {at }}$ depending on the reciprocal lattice parameters $a^{*}, b^{*}$, and $c^{*}$, and unit cell indices in reciprocal space $h, k, l$, as $T^{\text {at }}$ $=\exp \left[-2 \pi^{2}\left(U_{11}^{\text {at }} h^{2} a^{* 2}+U_{22}^{\text {at }} k^{2} b^{* 2}+U_{33}^{\text {at }} l^{2} c^{* 2}+2 U_{12}^{\text {at }} h a^{*} k b^{*}+2 U_{23}^{\text {at }} k b^{*} l c^{*}+U_{31}^{\text {at }} l c^{*} h a^{*}\right)\right]$.

\begin{tabular}{|c|c|c|c|c|c|c|}
\hline Atom & $U_{11}$ & $U_{22}$ & $U_{33}$ & $U_{23}$ & $U_{13}$ & $U_{12}$ \\
\hline \multirow[t]{2}{*}{$\mathrm{K}(1)$} & $0.0027(4)$ & $0.0018(4)$ & $0.0020(4)$ & 0.0000 & $0.0003(3)$ & 0.0000 \\
\hline & $0.035(1)$ & $0.0263(8)$ & $0.0325(9)$ & 0.0000 & $0.0122(7)$ & 0.0000 \\
\hline \multirow[t]{2}{*}{$\mathrm{C}(1)$} & $0.00117(19)$ & $0.00268(19)$ & $0.0023(2)$ & $0.00024(13)$ & $0.00039(14)$ & $0.00003(13)$ \\
\hline & $0.0297(4)$ & $0.0329(4)$ & $0.0299(3)$ & $0.0029(3)$ & $0.0078(3)$ & $-0.0022(3)$ \\
\hline \multirow[t]{2}{*}{$\mathrm{C}(2)$} & $0.00157(19)$ & $0.00238(19)$ & $0.0023(2)$ & $0.00055(14)$ & $0.00060(14)$ & $0.00064(13)$ \\
\hline & $0.0258(3)$ & $0.0272(3)$ & $0.0249(3)$ & $0.0045(3)$ & $0.0043(2)$ & $0.0005(3)$ \\
\hline \multirow[t]{2}{*}{$\mathrm{O}(1)$} & $0.0038(2)$ & $0.0048(2)$ & $0.0035(2)$ & $0.00241(17)$ & $0.00120(17)$ & $0.00108(15)$ \\
\hline & $0.0360(5)$ & $0.0341(4)$ & $0.0307(4)$ & $0.0115(4)$ & $0.0088(3)$ & $0.0042(4)$ \\
\hline \multirow[t]{2}{*}{$\mathrm{O}(2)$} & $0.0033(2)$ & $0.0060(2)$ & $0.0047(2)$ & $0.00268(17)$ & $0.00194(17)$ & $0.00312(16)$ \\
\hline & $0.0376(5)$ & $0.0507(7)$ & $0.0418(5)$ & $0.0194(5)$ & $0.0129(4)$ & $0.0180(4)$ \\
\hline \multirow[t]{2}{*}{$\mathrm{F}(1)$} & $0.0049(2)$ & $0.0048(2)$ & $0.0054(2)$ & $0.00042(18)$ & $0.00072(18)$ & $-0.00222(17)$ \\
\hline & $0.0523(8)$ & $0.0457(7)$ & $0.0597(8)$ & $0.0074(6)$ & $0.0150(6)$ & $-0.0195(6)$ \\
\hline \multirow[t]{2}{*}{$\mathrm{F}(2)$} & $0.0027(2)$ & $0.0054(2)$ & $0.0057(2)$ & $0.00058(18)$ & $0.00076(18)$ & $0.00219(17)$ \\
\hline & $0.0353(6)$ & $0.0579(8)$ & $0.0557(7)$ & $0.0057(6)$ & $0.0135(5)$ & $0.0141(5)$ \\
\hline \multirow[t]{2}{*}{$\mathrm{F}(3)$} & $0.0054(2)$ & $0.0057(2)$ & $0.0025(2)$ & $-0.00028(17)$ & $0.00020(17)$ & $-0.00006(17)$ \\
\hline & $0.0608(8)$ & $0.0535(7)$ & $0.0285(5)$ & $-0.0032(5)$ & $0.0094(5)$ & $0.0005(6)$ \\
\hline \multirow[t]{2}{*}{$\mathrm{H}(1)$} & $0.0177(7)$ & $0.0158(8)$ & $0.0160(7)$ & $0.0034(6)$ & $0.0023(6)$ & $0.0029(6)$ \\
\hline & $0.0539(17)$ & $0.0577(18)$ & $0.0582(17)$ & $0.0199(15)$ & $0.0034(13)$ & $0.0152(14)$ \\
\hline
\end{tabular}


TABLE V. Thermal parameters in $\AA^{2}$ units for $\mathrm{CsH}\left(\mathrm{CF}_{3} \mathrm{COO}\right)_{2}$ at $14 \mathrm{~K}$ (first lines) and $298 \mathrm{~K}$ (second lines). The variance for the last digit is given in parentheses. See the caption of Table IV.

\begin{tabular}{lllllll}
\hline \hline Atom & \multicolumn{1}{c}{$U_{11}$} & \multicolumn{1}{c}{$U_{22}$} & \multicolumn{1}{c}{$U_{33}$} & \multicolumn{1}{c}{$U_{23}$} & \multicolumn{1}{c}{$U_{13}$} & $U_{12}$ \\
\hline $\mathrm{Cs}(1)$ & $0.0009(3)$ & $0.0015(3)$ & $0.0019(3)$ & 0.0000 & $0.0009(2)$ & 0.0000 \\
& $0.0319(6)$ & $0.0359(7)$ & $0.0526(9)$ & 0.0000 & $0.0244(6)$ & 0.0000 \\
$\mathrm{~F}(1)$ & $0.0048(2)$ & $0.0069(3)$ & $0.0061(2)$ & $-0.00185(18)$ & $0.00239(17)$ & $0.00167(19)$ \\
& $0.0614(8)$ & $0.069(1)$ & $0.068(1)$ & $-0.0250(8)$ & $0.0275(7)$ & $0.0110(8)$ \\
$\mathrm{F}(2)$ & $0.0095(2)$ & $0.0082(3)$ & $0.0030(2)$ & $0.00077(19)$ & $0.00246(17)$ & $0.0006(2)$ \\
& $0.0959(13)$ & $0.0907(14)$ & $0.0369(7)$ & $0.0065(8)$ & $0.0199(8)$ & $0.0014(12)$ \\
$\mathrm{F}(3)$ & $0.0074(2)$ & $0.0047(3)$ & $0.0068(3)$ & $-0.00216(19)$ & $0.00288(18)$ & $-0.00294(18)$ \\
& $0.0837(12)$ & $0.0581(9)$ & $0.0777(12)$ & $-0.0291(9)$ & $0.0342(9)$ & $-0.0324(9)$ \\
$\mathrm{O}(1)$ & $0.0038(2)$ & $0.0055(2)$ & $0.0071(2)$ & $-0.00185(18)$ & $0.00373(16)$ & $-0.00097(18)$ \\
& $0.0344(5)$ & $0.0458(6)$ & $0.0592(7)$ & $-0.0061(6)$ & $0.0245(5)$ & $-0.0035(5)$ \\
$\mathrm{O}(2)$ & $0.0029(2)$ & $0.0039(2)$ & $0.0068(2)$ & $-0.00313(18)$ & $0.00219(16)$ & $-0.00094(17)$ \\
& $0.0305(4)$ & $0.0407(6)$ & $0.0634(7)$ & $-0.0202(6)$ & $0.0202(5)$ & $-0.0076(4)$ \\
$\mathrm{C}(1)$ & $0.00369(17)$ & $0.0030(2)$ & $0.00295(18)$ & $-0.00084(14)$ & $0.00151(13)$ & $0.00004(15)$ \\
& $0.0448(5)$ & $0.0407(5)$ & $0.0346(4)$ & $-0.0067(4)$ & $0.0148(3)$ & $-0.0051(4)$ \\
$\mathrm{C}(2)$ & $0.00267(18)$ & $0.00290(19)$ & $0.00315(18)$ & $-0.00089(15)$ & $0.00183(13)$ & $-0.00025(14)$ \\
& $0.0285(3)$ & $0.0284(3)$ & $0.0370(4)$ & $-0.0012(3)$ & $0.0130(3)$ & $-0.0011(3)$ \\
$\mathrm{H}(1)$ & $0.0131(6)$ & $0.0176(8)$ & $0.0185(7)$ & $0.0006(7)$ & $0.0053(5)$ & $-0.0011(6)$ \\
& $0.0403(13)$ & $0.0498(16)$ & $0.088(2)$ & $-0.0059(17)$ & $0.0247(14)$ & $-0.0011(12)$ \\
\hline \hline & & & & & &
\end{tabular}

scattering function, on the other hand. ${ }^{48}$ The incoherent cross section is rather large for protons $\left(\sigma_{H} \approx 80 \mathrm{~b}\right)$ and negligibly small for all other nuclei under consideration. The scattering function $S(\mathbf{Q}, \nu)$ depends on the neutron momentum transfer vector $\mathbf{Q}=\mathbf{k}_{0}-\mathbf{k}_{f}$, defined by the initial, $\mathbf{k}_{0}$, and the final, $\mathbf{k}_{f}$, wave vectors, and on the energy transfer $h \nu$. With the time focussed crystal analyzer (TFXA) spectrometer utilized in Ref. 44, only scattering events corresponding to the maximum of the scattering function for harmonic bare protons are counted (see Appendix).

The previously reported INS spectra of the two salts are very similar. ${ }^{44}$ The $\gamma \mathrm{H}$ modes give narrow single bands at $\approx 1225 \mathrm{~cm}^{-1}$ (KTFA) or $\approx 1260 \mathrm{~cm}^{-1}$ (CTFA) and overtones at $\approx 2450 \mathrm{~cm}^{-1}$ (KTFA) or $\approx 2515 \mathrm{~cm}^{-1}$ (CTFA), consistent with quasiharmonic oscillators. The intensity ratios $I_{0 \rightarrow 2} / I_{0 \rightarrow 1}$ accord with an oscillator mass of $\approx 1 \mathrm{amu}$. The full widths at half maximum (FWHMs) of $\approx 50 \mathrm{~cm}^{-1}$ are representative of the density of states, convoluted with the spectrometer resolution $(\Delta \nu / \nu \approx 0.02)$, that is, $\Delta \nu$ $\approx 25 \mathrm{~cm}^{-1}$ at $1250 \mathrm{~cm}^{-1}$. The upper bound for the frequency dispersion (FWHM $-\Delta \nu \approx 25 \mathrm{~cm}^{-1}$ ) accords with rather small coupling terms between protons. Similarly, the $\delta \mathrm{H}$ modes show single bands at $\approx 1600 \mathrm{~cm}^{-1}$ (FWHM $\approx 70 \mathrm{~cm}^{-1}$ ) for both salts. For KTFA, the weak overtone at $\approx 3200 \mathrm{~cm}^{-1}$ is also consistent with a quasiharmonic oscillator with $m \approx 1 \mathrm{amu}$. (The overtone is not clearly observed for CTFA, presumably because of larger statistical errors.)

In marked contrast to the bending modes, the stretching shows broad profiles, composed of partially resolved subbands between 500 and $1100 \mathrm{~cm}^{-1}$, which cannot be rationalized with a quasiharmonic model (see Fig. 4). Tentative decompositions with Gaussian components are very similar for the two salts (Fig. 4 and Table VI). Only one component at $\approx 850 \mathrm{~cm}^{-1}$ for CTFA is not visible for KTFA. Such similar profiles for quite different crystal structures suggest that the crystal field is not the main band splitting factor. Further information can be sorted out of the infrared and Raman spectra.

In the infrared, the $\gamma \mathrm{H}$ are rather weak and the $\delta \mathrm{H}$ are practically invisible. The spectra are dominated by narrow bands, due to TFA entities, superimposed to the very broad stretching profiles. The maxima of intensity at $\approx 800 \mathrm{~cm}^{-1}$ coincide with those observed with INS. Above $1000 \mathrm{~cm}^{-1}$, there are long tails of continuous intensity, extending up to at least $2000 \mathrm{~cm}^{-1}$, which are not observed with INS. The broader profiles observed in the infrared, at the Brillouinzone center, compared to INS, for the whole density of states, confirm that dispersion is not the main band shaping mechanism. In fact, weak dynamical coupling is in line with the rather large proton-proton distances in the crystals. (The shortest distances are $4.34 \AA$ for KTFA and $6.72 \AA$ for CTFA.)

The Raman spectra are exclusively due to the TFA entities, while proton modes are invisible. For KTFA, the strong Raman bands at $850-854 \mathrm{~cm}^{-1}$, analogous to those observed for CTFA, ${ }^{44}$ suggest that the INS counterpart should exist, although it is not resolved.

The assignment scheme in Table VII is based on the contrast of intensities measured with INS, infrared, and Raman. (The dubious component above $1000 \mathrm{~cm}^{-1}$ is ignored.) INS components visible in Raman are assigned to TFA modes acquiring INS intensity via resonance with proton states (see below). The other subbands are associated with $\left|\nu_{a}\right\rangle_{n}$ states. Owing to the spectrometer resolution rang- 

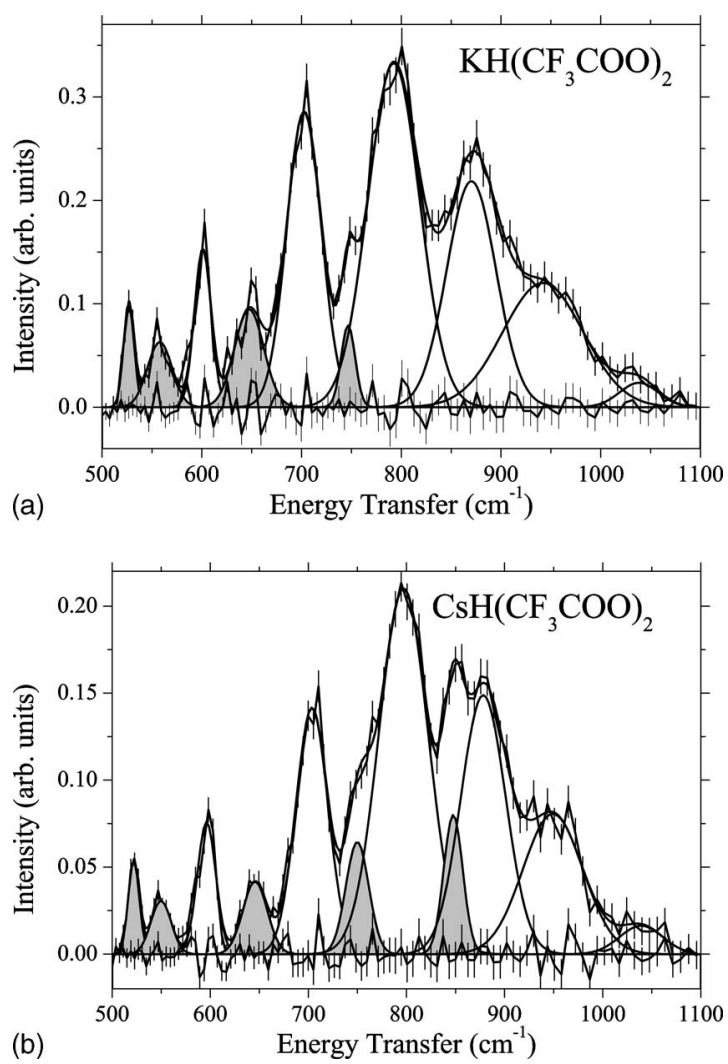

FIG. 4. Inelastic neutron scattering spectra and band decomposition into Gaussian profiles in the $\nu_{a}$ region for $\mathrm{KH}\left(\mathrm{CF}_{3} \mathrm{COO}\right)_{2}$ and $\mathrm{CsH}\left(\mathrm{CF}_{3} \mathrm{COO}\right)_{2}$ at $20 \mathrm{~K}$. Only the filled components have counterparts in Raman (see text). The residual of the fit is compared to error bars.

ing from $\approx 10$ to $20 \mathrm{~cm}^{-1}$, the intrinsic bandwidths should be much less than the FWHMs in Table VII. Such narrow components are consistent with long-live stationary states.

\section{PROTON DYNAMICS}

Within the framework of the Born-Oppenheimer approximation, the vibrational Hamiltonian can be partitioned as

$$
\mathcal{H}_{v}=\mathcal{H}_{\mathrm{H}}+\mathcal{H}_{\mathrm{at}}+\mathcal{C}_{\text {Hat }},
$$

where $\mathcal{H}_{\mathrm{H}}$ and $\mathcal{H}_{\mathrm{at}}$ represent protons $\left(\mathrm{H}^{\oplus}\right)$ and heavy atoms, respectively, while $\mathcal{C}_{\text {Hat }}$ couples the subsystems. For OHO hydrogen bonds, the dominant coupling term between $\nu_{\mathrm{OH}}$ and $\nu_{\mathrm{OO}}$ can be evaluated from the slope $S=\Delta \nu_{\mathrm{OH}} / \Delta R_{\mathrm{OO}}$ of the empirical correlation $\nu_{\mathrm{OH}}\left(R_{\mathrm{OO}}\right) .{ }^{10,19}$ According to Novak, ${ }^{19}$ the slope increases continuously as $R_{\mathrm{OO}}$ decreases: $S \approx 1500 \mathrm{~cm}^{-1} \AA^{-1}$ for weak hydrogen bonds $\left(R_{\mathrm{OO}}>2.7 \AA\right)$, $S \approx 5000 \mathrm{~cm}^{-1} \AA^{-1}$ for intermediate hydrogen bonds $\left(R_{\mathrm{OO}} \approx 2.6-2.7 \AA\right)$, and $S \approx 12000 \mathrm{~cm}^{-1} \AA^{-1}$ for strong hydrogen bonds $\left(R_{\mathrm{OO}}<2.6 \AA\right)$. This strong coupling is widely recognized as an important band shaping factor. ${ }^{3,49-52}$

However, we argue that the slope for strong hydrogen bonds should not be extrapolated for VSHBs without caution because there is no known example of hydrogen bonds with $R_{\mathrm{OO}}$ significantly shorter than $\approx 2.4 \AA$ and stretching frequency much lower than $\approx 500 \mathrm{~cm}^{-1}$. [For the time being, we put aside the dubious assignment at $371 \mathrm{~cm}^{-1}$ for $4 \mathrm{CTH}$ (Ref. 22) (see above).] We posit that the stretching frequency reaches a minimum value for VSHBs similar to those in TFAs or KHM, so the leading coupling term in Eq. (1) vanishes $(S \approx 0)$ and proton dynamics are only weakly coupled to oxygen atoms. In the zeroth-order approximation, the spectral profile is representative of the potential function along the stretching coordinate, say, $\mathcal{V}\left(x_{a}\right)$, and weak coupling terms with other degrees of freedom can be treated as perturbations. (Note that the potential function for KHM was determined on this basis. ${ }^{27}$ )

\section{A. Resonance}

Weak coupling terms account for INS intensities of the otherwise invisible TFA modes. Within the framework of the adiabatic separation of $\mathcal{H}_{\mathrm{H}}$ and $\mathcal{H}_{\mathrm{at}}{ }^{53,54}$ the ground-state vector can be written as

$$
\left|x_{a}\right\rangle_{0}|y\rangle_{0}|z\rangle_{0} \underset{i}{\otimes}\left|X_{i}\right\rangle_{0}\left|Y_{i}\right\rangle_{0}\left|Z_{i}\right\rangle_{0}
$$

where $x_{a}, y, z$ are proton coordinates, while $X_{i}, Y_{i}, Z_{i}$, are normal coordinates for heavy atoms. When an excited proton state, say, $\left|x_{a}\right\rangle_{n}$, is close enough to a heavy atom state, $\left|A_{i}\right\rangle_{\mathrm{Ni}}$

TABLE VI. Gaussian decomposition of the INS spectra of $\mathrm{KH}\left(\mathrm{CF}_{3} \mathrm{COO}\right)_{2}(\mathrm{KTFA})$ and $\mathrm{CsH}\left(\mathrm{CF}_{3} \mathrm{COO}\right)_{2}$

\begin{tabular}{|c|c|c|c|c|c|c|c|}
\hline \multirow[b]{2}{*}{ Peak } & \multicolumn{2}{|c|}{ Gravity center $\left(\mathrm{cm}^{-1}\right)$} & \multicolumn{2}{|c|}{ FWHM $\left(\mathrm{cm}^{-1}\right)$} & \multicolumn{2}{|c|}{ Area $(\%)$} & \multirow[b]{2}{*}{ Observed } \\
\hline & KTFA & CTFA & KTFA & CTFA & KTFA & CTFA & \\
\hline 1 & 527 & 522 & 12 & 13 & 1.8 & 1.8 & INS, R \\
\hline 2 & 559 & 550 & 29 & 24 & 2.7 & 1.8 & INS, R, IR \\
\hline 3 & 601 & 596 & 20 & 22 & 4.5 & 4.2 & INS \\
\hline 4 & 647 & 646 & 35 & 29 & 4.8 & 3.0 & INS, R, IR \\
\hline 5 & 703 & 704 & 41 & 39 & 17.2 & 13.5 & INS \\
\hline 6 & 747 & 749 & 17 & 28 & 1.9 & 4.4 & INS, R, IR \\
\hline 7 & 792 & 797 & 61 & 59 & 29.9 & 30.6 & INS \\
\hline 8 & $\cdots$ & 848 & $\cdots$ & 23 & $\cdots$ & 4.5 & INS, R, IR \\
\hline 9 & 870 & 878 & 58 & 54 & 18.8 & 19.8 & INS \\
\hline 10 & 942 & 949 & 95 & 72 & 16.8 & 14.3 & INS \\
\hline 11 & 1038 & 1039 & 46 & 52 & 1.6 & 2.1 & INS \\
\hline
\end{tabular}
(CTFA) in the $\mathrm{OH}$ stretching region (see Fig. 4). FWHM: Full width at half maximum. IR: Infrared. R: Raman. 
TABLE VII. Observed and calculated $\mathrm{OH}$ stretching frequencies and INS relative intensities for $\mathrm{KH}\left(\mathrm{CF}_{3} \mathrm{COO}\right)_{2}$ for the potential function $V=-185.074 x+122.598 x^{2}-10.506 x^{3}-1232.04 \exp \left(-28.149 x^{2}\right)$. $V$ and $x$ are in $\mathrm{cm}^{-1}$ and $\AA$ units, respectively.

\begin{tabular}{ccccccccc}
\hline \hline & \multicolumn{3}{c}{ Observation } & & & \multicolumn{3}{c}{ Calculation } \\
\cline { 2 - 4 } \cline { 7 - 8 } Transitions & $\begin{array}{c}h \nu \\
\left(\mathrm{cm}^{-1}\right)\end{array}$ & $\begin{array}{c}Q \\
\left(\AA^{-1}\right)\end{array}$ & $\begin{array}{c}\text { Int. } \\
(\text { a.u. })\end{array}$ & Grouping & & $\begin{array}{c}h \nu \\
\left(\mathrm{cm}^{-1}\right)\end{array}$ & $\begin{array}{c}Q \\
\left(\AA^{-1}\right)\end{array}$ & $\begin{array}{c}\text { Int. } \\
(\text { a.u. })\end{array}$ \\
\hline $0 \rightarrow 1$ & 601 & 6.0 & 0.30 & $1+2+3$ & 614 & 6.1 & 0.38 \\
$0 \rightarrow 2$ & 703 & 6.5 & 0.80 & $4+5+6$ & 701 & 6.5 & 0.93 \\
$0 \rightarrow 3$ & 792 & 6.9 & 1.00 & 7 & 787 & 6.9 & 1.00 \\
$0 \rightarrow 4$ & 870 & 7.2 & 0.63 & 8 & 872 & 7.2 & 0.59 \\
$0 \rightarrow 5$ & 942 & 7.6 & 0.56 & 9 & 953 & 7.5 & 0.37 \\
\hline \hline
\end{tabular}

$\left(A_{i}=X_{i}, Y_{i}, Z_{i}\right)$, weak coupling terms lead to state mixing (resonance). Within the framework of first order perturbation theory, the energies of the uncoupled states are shifted apart and the mixed vector states can be written as

$$
\begin{aligned}
& |1\rangle=\alpha\left|x_{a}\right\rangle_{n}+\beta\left|A_{i}\right\rangle_{N i}, \\
& |2\rangle=-\beta\left|x_{a}\right\rangle_{n}+\alpha\left|A_{i}\right\rangle_{N i},
\end{aligned}
$$

with $\alpha^{2}+\beta^{2}=1$. The INS intensity of the transition to the unperturbed state, $\left|x_{a}\right\rangle_{0} \rightarrow\left|x_{a}\right\rangle_{n}$, is distributed among transitions $\left|x_{a}\right\rangle_{0} \rightarrow|1\rangle$ and $\left|x_{a}\right\rangle_{0} \rightarrow|2\rangle$ with relative intensities proportional to $\alpha^{2}$ and $\beta^{2}$, respectively. Such resonances are more likely to occur among the numerous $\left|x_{a}\right\rangle_{n}$ states than with the quasiharmonic bending states. In addition, more than two states can be eventually mixed, but we do not need to elaborate any further, as our purpose is limited to a qualitative interpretation.

The INS intensities of the components at 527 and $559 \mathrm{~cm}^{-1}$ in Table VI are, thus, attributed to resonances with TFA modes. These intensities are added to the $0 \rightarrow 1$ transition (Table VII) and the resonance induced frequency shift is neglected. Similarly, the intensity of the $0 \rightarrow 2$ transition is the sum of intensities for components 4-6. This grouping is likely not unique and possible resonances with other TFA modes below $500 \mathrm{~cm}^{-1}$ are ignored. This leads to unavoidable uncertainties for relative intensities and unperturbed levels. A very similar assignment scheme could be proposed for CTFA.

\section{B. Potential function}

By analogy with the funnel-shaped potential of KHM, $\mathcal{V}\left(x_{a}\right)$ (Fig. 5) is represented with a narrow Gaussian well, imposed by the $0 \rightarrow 1$ transition at $601 \mathrm{~cm}^{-1}$, and a broad quasiharmonic potential, shifted off center, consistent with the energy-level spacing $\left(\lesssim 100 \mathrm{~cm}^{-1}\right)$ for higher excited states and with the observed relative intensities as

$$
\mathcal{V}\left(x_{a}\right)=-a x_{a}+b x_{a}^{2}-c x_{a}^{3}-d \exp \left(-f x_{a}^{2}\right) .
$$

In addition to the Gaussian and harmonic wells, the linear term is crucial to adjusting relative intensities, and the cubic term accounts for anharmonic corrections to the upper energy levels. For the sake of definiteness, the number of parameters is set equal to the number of observed transitions. Note that this model potential cannot account for the con- tinuum of infrared intensity that could be due to unbound states.

Eigenstates were calculated with the variational method (see Appendix) and the coefficients were adjusted via least squares fitting exercises. The maximum deviation of $\approx 2 \%$ (Table VII) is comparable to the spectrometer resolution and within the bandwidths. The calculated intensities are in qualitative agreement with observations, so the eigenfunctions should be reasonably well calculated. Needless to say, the potential shape is imposed by the assignment scheme, so additional terms in Eq. (4), alternative grouping of intensities, or minor corrections for the unperturbed levels should be of no consequence to the overall shape.

\section{Discussion}

The wave function in the ground state is practically symmetrical with respect to the center, in accordance with the crystal structure. In addition, the mean-square amplitude (the variance of the Gaussian-like squared wave function, $u_{0 a}^{2} \approx 0.02 \AA^{2}$ ) is comparable to the thermal parameters at low temperatures (Table IV). It is worthy of note that a double well with a barrier height below the ground state would increase $u_{0 a}^{2}$ beyond the limit imposed by the thermal factor. At room temperature, the population of excited states

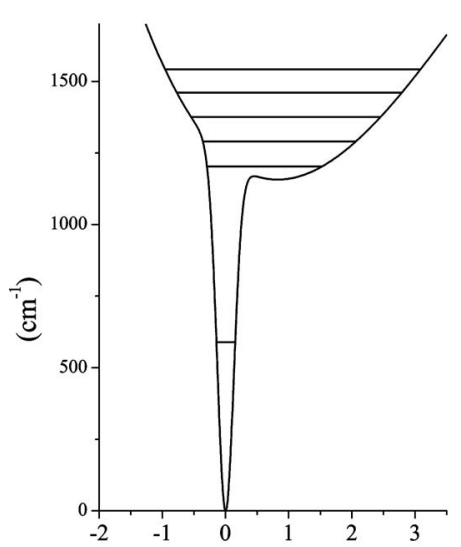

$(\AA)$

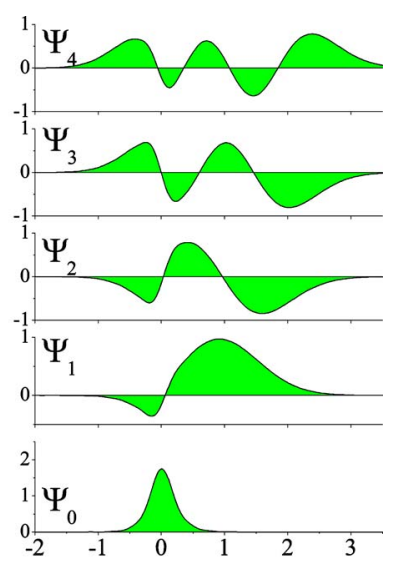

$(\AA)$
FIG. 5. (Color online) Left: Calculated potential and energy levels for $\quad \mathrm{KH}\left(\mathrm{CF}_{3} \mathrm{COO}\right)_{2} . \quad V=-185.074 x+122.598 x^{2}-10.506 x^{3}-1232.04$ $\times \exp \left(-28.149 x^{2}\right)$, with $V$ and $x$ in $\mathrm{cm}^{-1}$ and $\AA$ units, respectively. Right: Calculated wave functions. 


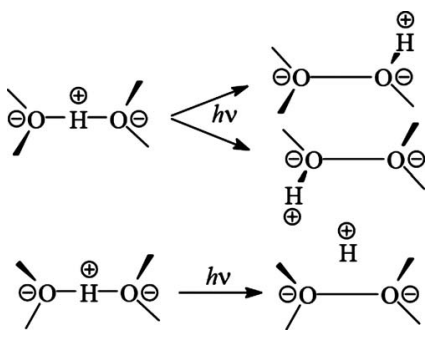

FIG. 6. Schematic representations of SSHBs in trifluoroacetate dimers (top) and hydrogen maleate (bottom), in the ground state (left), and in excited vibrational states (right). The wedge bonds represent lone pair orbitals (see text).

$(\approx 5 \%)$ should give a diffuse probability density too weak to be visible with the precision of neutron diffraction.

In the excited states, the spatial extension of the wave functions is much greater than $R_{\mathrm{OO}}$, so the proton is no longer confined between the $\mathrm{O}$ atoms. For KHM, it was speculated that the hydrogen bond could be broken at a rather modest energy cost, thanks to the internal strain of the ring. ${ }^{27}$ However, this view was not confirmed with density functional theory calculations. ${ }^{23}$ Furthermore, there is no internal strain for intermolecular SSHBs, so this option must be abandoned.

Upon the assumption that the bond is not broken by vibrational excitations, the mean position $\left\langle x_{a}^{2}\right\rangle^{1 / 2} \approx 1 \AA$ in the excited states of KTFA suggests that the proton is localized around one of the two oxygens. It seems to be weakly bound to the lone pairs via the overlap between vibrational wave functions and electron orbitals. Similarly, the delocalized wave functions in the upper symmetrical well of KHM (Fig. 1) could overlap the lone pairs of the two oxygens. The schemes presented in Fig. 6 suggest a correlation between the potential asymmetry/symmetry and the spatial orientation of the lone pairs. For intermolecular hydrogen bonds, lone pairs pointing to opposite directions lead to localization of the excited proton states around either of the two oxygens, hence an asymmetric potential. Alternatively, lone pairs in the plane of the maleate ring lead to delocalization over the two oxygens and a symmetric potential.

If oxygens are thought of as hard spheres, the spread out of the wave functions along $x_{a}$ is possible if large displacements occur simultaneously perpendicular to the $\mathrm{OO}$ direction. Vibrational spectra do not provide any definite information on such displacements, except that there is no evidence of any rotation of the proton coordinates in the excited states. Otherwise, one should observe some mixing of the stretching and bending modes. Theoretical modeling should consider very large proton displacements in multidimensions.

In the ground state, hydrogen bonds can be conceived of as bare protons sitting at the center: ${ }^{\ominus} \mathrm{O}-\mathrm{H}^{\oplus}-\mathrm{O}^{\ominus}$. The binding energy of $\mathrm{H}^{\oplus}$ to the local maximum of the density of negative charges corresponds to the depth of the central well minus the zero-point energy, namely, $\approx 600 \mathrm{~cm}^{-1}$ for TFAs or $\approx 500 \mathrm{~cm}^{-1}$ for KHM. They are marginal compared to the total hydrogen bond energy. This ionic scheme is at variance with the resonance $\mathrm{O}-\mathrm{H} \cdots \mathrm{O}^{\ominus} \leftrightarrow{ }^{\ominus} \mathrm{O} \cdots \mathrm{H}-\mathrm{O}$ advocated by Gilli et al. in order to stress the covalent character of the OH bond. ${ }^{8,23}$
For the sake of preserving the center of symmetry, excited vibrational states of the TFAs must be represented by mixtures, with equal probabilities, of nonoverlapping degenerate states $\left|{ }^{\ominus} \mathrm{O}-\mathrm{O}^{\ominus} \mathrm{H}^{\oplus}\right\rangle_{n}$ and $\left|{ }^{\oplus} \mathrm{H}^{\ominus} \mathrm{O}-\mathrm{O}^{\ominus}\right\rangle_{n}$, located on either sides of the $\mathrm{OO}$ bond (Fig. 6). These states can be thought of as "localized proton orbitals." For KHM, excited vibrational states are represented by symmetric and antisymmetric superposition states, $2^{-1 / 2}\left[\left|{ }^{\ominus} \mathrm{O}-\mathrm{O}^{\ominus} \mathrm{H}^{\oplus}\right\rangle \pm\left|{ }^{\oplus} \mathrm{H}^{\ominus} \mathrm{O}-\mathrm{O}^{\ominus}\right\rangle\right]$, arising from the overlap between the localized orbitals. These states can be termed "delocalized proton orbitals." The rather small $|1\rangle-|2\rangle$ splitting of $30 \mathrm{~cm}^{-1}$ in Fig. 1, compared to the mean level spacing greater than $100 \mathrm{~cm}^{-1}$, suggests proton "tunneling" between the shallow minima created by lone pairs. It is not clear as to whether these delocalized states contribute to the binding energy. In any case, this contribution should be marginal.

In the excited states, the charge compensating proton is apparently no longer involved in the bonding, so we speculate that the "bare" ${ }^{\ominus} \mathrm{O}-\mathrm{O}^{\ominus}$ bond is intrinsically stable. To suppose that it is covalent in nature is not irrelevant since binding energies for SSHBs are on the scale of those for peroxides $(\mathrm{O}-\mathrm{O} \approx 1.48 \AA)$, found in the range of $45-35 \mathrm{kcal} \mathrm{mol}^{-1}$. ${ }^{55}$ Needless to say, this comparison is not a proof that ${ }^{\ominus} \mathrm{O}-\mathrm{O}^{\ominus}$ bonds are the longest covalent bonds ever known. This interpretation deserves further theoretical investigations.

The funnel potentials suggest that the total binding energy can be partitioned in to two parts: The binding energy for protons, the "hydrogen bonding" contribution and the "covalent bonding" energy for the bare ${ }^{\ominus} \mathrm{O}-\mathrm{O}^{\ominus}$ bond. Accordingly, SSHBs are essentially covalent bonds, with a marginal hydrogen bond character. ${ }^{56,57}$ If we suppose that for shorter $R_{\mathrm{OO}}$, the binding energy of $\mathrm{H}^{\oplus}$ vanishes, then SSHBs should be close to the physical limit for the existence of hydrogen bonds. Conversely, we extrapolate that as $R_{\mathrm{OO}}$ increases, the hydrogen bond character increases while the covalent bonding decreases, so that the total binding energy decreases. For the sake of continuity near the dissociation threshold, the $\mathrm{O} \cdots \mathrm{H}-\mathrm{O}$ scheme emphasizing the covalent nature of the $\mathrm{OH}$ bond and long-range dipolar interactions should supersede the "ionic" scheme.

\section{CONCLUSION}

Very short hydrogen bonds linking trifluoroacetate entities in crystals are symmetrical at room and cryogenic temperatures. The thermal ellipsoids exclude a double well for protons, within the limitations imposed by quantum mechanics. The contrasts of intensities (infrared, Raman, and INS) allow us to establish an assignment scheme for the spectral profiles.

We argue that bond lengths and proton stretching frequencies of SSHBs are close to the lower bounds at which the main coupling term between proton and oxygen atoms goes to zero. Proton dynamics are largely insulated from the lattice and weak coupling terms can be treated as perturbations. Vibrational spectra are consistent with funnel-shaped potentials that could be distinctive of very strong hydrogen 
bonds. Protons in excited vibrational states are delocalized over the lone pairs of oxygen atoms. These states suggest a marked covalent character of $-\mathrm{O}^{\ominus}-\mathrm{O}^{\ominus}$ bonds and weak electrostatic interaction with the ionic proton.

Potential functions consistent with energy levels, profiles of intensity, and thermal ellipsoids are overdetermined. They enlighten on unforeseen dynamics and electronic structures suggesting that there is not yet a clear understanding of the nature of the hydrogen bond at the fundamental level of quantum mechanics. We propose a comprehensive rationale accounting for similarities (funnel potentials) and differences (symmetry/asymmetry) of the three best studied SSHBs. However, the adequacy of a model to observations, as good as it can be, is not a definite proof that this model is unique. The interpretation advocated in this work should be confronted with complementary experiments, for example, solid-state NMR, and theoretical investigations.

\section{APPENDIX: CALCULATION OF ENERGY LEVELS, WAVE FUNCTIONS, AND INS INTENSITIES}

The variational method is appropriate to determine analytical potential functions fitting any given energy level scheme. The expansion of the eigenfunctions with harmonic basis sets allows us to calculate all matrix elements of interest for vibrational spectroscopy. In this Appendix, we gather the formulas to resolve the Schrödinger equation for a dimensionless particle with mass $m$ experiencing a potential $V(x)$ along the $x$ coordinate,

$$
-\frac{\hbar^{2}}{2 m} \frac{\mathrm{d}^{2} \Psi}{\mathrm{d} x^{2}}+V(x) \Psi=E \Psi
$$

with

$$
V(x)=V_{p}(x)+V_{G}(x)=\sum_{l=1}^{6} v_{l} x^{l}+\sum_{l=1}^{3} a_{l} \exp \left(-b_{l} x^{2}\right),
$$

where $V_{p}$ is the polynomial and $V_{G}$ is the sum of three Gaussians, to allow for a rich variety of potential shapes. We also propose a straightforward method to test the accuracy of the eigenfunctions.

In order to construct a basis set, let $\nu_{0}=\omega_{0} /(2 \pi)$ be a frequency and consider the dimensionless variables $\xi=\alpha x$, with $\alpha=\sqrt{m \omega_{0} / \hbar}, \tilde{V}=V /\left(\hbar \omega_{0}\right)$, and $\widetilde{E}=E /\left(\hbar \omega_{0}\right)$. Then,

$$
\widetilde{V}_{p}=\sum_{l=1}^{6} \widetilde{v}_{l} \xi^{l},
$$

with

$$
\widetilde{v}_{l}=v_{l} /\left(\hbar \omega_{0} \alpha^{l}\right) .
$$

Here, the scaled energies are such that the level spacing of the harmonic oscillator is 1 instead of 4 in Ref. 56. The coefficients $v_{l}$, divided by a factor of 2 compared to Ref. 56, are such that the potential energy of the harmonic oscillator $\tilde{V}=\xi^{2} / 2$ corresponds to $\widetilde{v}_{l}=1 / 2 \delta_{l, 2} \quad$ (where $\delta_{l, 2}$ is the Kronecker symbol). Then, $\alpha=\alpha_{0} \sqrt{\widetilde{\nu}_{0} m}$ can be expressed in $\AA^{-1}$ units with $\alpha_{0}=0.17273$ and $\widetilde{\nu}_{0}=\omega_{0} /(2 \pi c)$ in $\mathrm{cm}^{-1}$ units.

In the new variables, Eq. (A1) reads

$$
\hat{h} \psi=-\frac{1}{2} \frac{\mathrm{d}^{2} \psi}{\mathrm{d} \xi^{2}}+\widetilde{V} \psi=\widetilde{E} \psi,
$$

with $\psi=\sqrt{\alpha} \Psi$ normalized with respect to $\xi$. For a harmonic oscillator, the normalized solutions of Eq. (A5) are

$$
u_{n}(\xi)=\left(2^{n} n ! \sqrt{\pi}\right)^{-1 / 2} H_{n}(\xi) \exp \left(-\xi^{2} / 2\right), \quad n=0,1, \ldots,
$$

$\left\{H_{n}\right\}$ being the Hermite polynomials. The functions $\left\{u_{n}\right\}$ form a suitable orthonormal basis set for bound states. For numerical calculations, the dimension $N$ of the basis has to be finite. We found $N=60$ is suitable to calculate the ten lowest energy levels with good accuracy (see below). The matrix elements of $\hat{h}$ in Eq. (A5) are

$$
\hat{h}_{n, m}=\langle n|\hat{h}| m\rangle=\int_{-\infty}^{\infty} u_{n}(\xi) \hat{h} u_{m}(\xi) \mathrm{d} \xi .
$$

The matrix elements for a sixth order polynomial potential or a Gaussian potential can be found in Refs. 56 and 57, respectively. With the alternative definition of the scaling factors, the algorithms for the polynomial form are.

$$
\begin{aligned}
\hat{h}_{n, n}^{p}= & \left(n+\frac{1}{2}\right)\left(\frac{1}{2}+\widetilde{v}_{2}\right)+\frac{3}{4}\left(2 n^{2}+2 n+1\right) \widetilde{v}_{4} \\
& +\frac{1}{8}\left(20 n^{3}+30 n^{2}+40 n+15\right) \widetilde{v}_{6},
\end{aligned}
$$

$$
\hat{h}_{n, n-1}^{p}=\frac{1}{2} \sqrt{2 n}\left(\widetilde{v}_{1}+\frac{3}{2} n \widetilde{v}_{3}+\frac{5}{4}\left(2 n^{2}+1\right) \widetilde{v}_{5}\right),
$$

$$
\begin{aligned}
\hat{h}_{n, n-2}^{p}= & \frac{1}{2} \sqrt{n(n-1)}\left(-\frac{1}{2}+\widetilde{v}_{2}+(2 n-1) \widetilde{v}_{4}\right. \\
& \left.+\frac{15}{4}\left(n^{2}-n-1\right) \widetilde{v}_{6}\right),
\end{aligned}
$$

$$
\begin{aligned}
& \hat{h}_{n, n-3}^{p}=\frac{1}{2} \sqrt{\frac{n(n-1)(n-2)}{2}}\left(\widetilde{v}_{3}+\frac{5}{2}(n-1) \widetilde{v}_{5}\right), \\
& \hat{h}_{n, n-4}^{p}=\frac{1}{4} \sqrt{n(n-1)(n-2)(n-3)}\left(\widetilde{v}_{4}+\frac{3}{4}(2 n-3) \widetilde{v}_{6}\right),
\end{aligned}
$$$$
\hat{h}_{n, n-5}^{p}=\frac{1}{4} \sqrt{\frac{n(n-1)(n-2)(n-3)(n-4)}{2}} \widetilde{v}_{5},
$$

$$
\hat{h}_{n, n-6}^{p}=\frac{1}{8} \sqrt{n(n-1)(n-2)(n-3)(n-4)(n-5)} \widetilde{v}_{6} .
$$

All other matrix elements are zero except the symmetric ones: $\hat{h}_{n-l, n}^{p}=\hat{h}_{n, n-l}^{p}, l=1, \ldots, 6$.

For the Gaussian potential $W=a \exp \left(-b x^{2}\right)$, the scaled potential is $\widetilde{W}=\tilde{a} \exp \left(-\tilde{b} \xi^{2}\right)$, with $\widetilde{a}=a /\left(\hbar \omega_{0}\right)$ and $\tilde{b}=b / \alpha^{2}$. Matrix elements $\hat{W}_{n, m}$ in the subset $\left\{u_{n}\right\}_{n=0}^{N-1}$ are calculated according to Ref. 57 . The first row of a $(N, 2 N-1)$ auxiliary matrix $\hat{G}$ is 
$\hat{G}_{0, m}=\sqrt{\frac{m !}{2^{m}(1+\tilde{b})}} \frac{(-\theta)^{m / 2}}{(m / 2) !} \quad$ for $m($ even $)=0, \ldots, 2 N-1$,

with $\theta=\tilde{b} /(1+\tilde{b})$. The second row is

$$
\begin{aligned}
\hat{G}_{1, m}=\sqrt{m} \hat{G}_{0, m-1}+\sqrt{m+1} \hat{G}_{0, m+1} \\
\quad \text { for } m(\text { odd })=1, \ldots, 2 N-2 .
\end{aligned}
$$

Each successive row of index $n(2 \leqq n \leqq N-1)$ depends on the two previous ones as

$$
\begin{aligned}
\hat{G}_{n, m}= & 1 / \sqrt{n}\left(\sqrt{m} \hat{G}_{n-1, m-1}+\sqrt{m+1} \hat{G}_{n-1, m+1}\right. \\
& \left.-\sqrt{n-1} \hat{G}_{n-2, m}\right), \\
m=n, n & +2, n+4, \ldots, 2 N-n-1 .
\end{aligned}
$$

All elements not explicitly assigned are set to 0 . Let us redefine $\hat{G}$ as the $(N, N)$ square matrix corresponding to its first $N$ columns. The procedure above has led to an upper triangular matrix. The elements of the lower triangle are obtained by symmetry $\hat{G}(n, m)=\hat{G}(m, n)$ for all $n, m$, such that $m<n$. The $(N, N)$ matrix corresponding to $\widetilde{W}$ is $\hat{W}=\widetilde{a} \hat{G}(\widetilde{b})$, so the matrix $\hat{h}$ for the full potential [Eq. (A2)] is

$$
\hat{h}=\hat{h}^{p}+\sum_{l=1}^{3} \widetilde{a}_{l} \hat{G}\left(\widetilde{b}_{l}\right)
$$

If $\left\{\widetilde{E}_{n}\right\}_{n=0}^{N-1}$ are eigenvalues in increasing order and $\hat{C}$ is the $(N, N)$ matrix whose column $n$ is the normalized eigenvector corresponding to $\widetilde{E}_{n}$, the eigenfunctions are

$$
\psi_{n}(\xi)=\sum_{m=0}^{N-1} \hat{C}_{n, m} u_{m}(\xi)
$$

The eigenvalues and eigenfunctions in physical units are

$$
E_{n}=\hbar \omega_{0} \widetilde{E}_{n}
$$

and

$$
\Psi_{n}(x)=\sqrt{\alpha} \sum_{m=0}^{N-1} \hat{C}_{n, m} u_{m}(\alpha x) .
$$

The eigenfunctions are analytical functions whose derivatives are easily computed, so the accuracy can be checked by substitution in Eq. (A1). As a rule of thumb, the last coefficients of each series $\left\{\hat{C}_{n, m}\right\}_{m=0}^{N-1}$ (say, the last ten for $N=60$ ) have to be very small.

The parameter $\omega_{0}$ largely determines whether the truncated expansions of $\left\{\Psi_{n}\right\}$ are good approximations. As a rule of thumb, the exponential in Eq. (A6) should be small (say, $\sim \mathrm{e}^{-2}$ ) at the estimated limits for the the particle position. This leads to $\omega_{0} \approx 16 \hbar /\left(m \Delta x^{2}\right)$, where $\Delta x$ is the width of the classically allowed region or $\widetilde{\nu}_{0} \approx 16 /\left(m \alpha_{0}^{2} \Delta x^{2}\right)$ with $\widetilde{\nu}_{0}$ and $\Delta x$ in $\mathrm{cm}^{-1}$ and $\AA$ units, respectively. For a single minimum, this is obtained if $\hbar \omega_{0}$ is close to the first observed transition. In this case, $N=40$ is sufficient. For potentials composed of a narrow well and a shallow upper part, it is necessary to increase the size to $N=60$. The accuracy for the ten lower eigenvalues is largely within experimental errors and the accuracy of the eigenvectors is better than $1 \%$. Further increment of $N$ is unnecessary as numerical errors increase for higher powers of $x$.

The INS intensity for a transition $|0\rangle \rightarrow|n\rangle$ at energy $E_{n}$ is proportional to the scattering function ${ }^{48}$

$$
S(Q, E)=|\langle n|\exp (-i Q x)| 0\rangle|^{2} \delta\left(E-E_{n}\right) .
$$

For a spectrometer such as TFXA,${ }^{58}$ energy and momentum transfer, $E$ and $Q$, respectively, are correlated as

$$
E \approx 16.759 \times Q^{2},
$$

with $E$ and $Q$ in $\mathrm{cm}^{-1}$ and $\AA^{-1}$ units, respectively.

${ }^{1}$ G. Pimentel, C. McClellan, and A. Lester, The Hydrogen Bond (Freeman, San Francisco, 1960).

${ }^{2}$ S. N. Vinogradov and R. H. Linnell, Hydrogen Bonding (Van NostrandReinhold, New York, 1971).

${ }^{3}$ P. Schuster, G. Zundel, and C. Sandorfy, The Hydrogen Bond. Recent Developments in Theory and Experiments (North-Holland, Amsterdam, 1976), Vols. 1-3.

${ }^{4}$ G. A. Jeffrey and W. Saenger, Hydrogen Bonding in Biological Structures (Springer-Verlag, Berlin, 1991).

${ }^{5}$ S. Scheiner, Hydrogen Bonding: A Theoretical Perspective (Oxford University Press, Oxford, 1997).

${ }^{6}$ A. N. Baker, Jr., J. Chem. Phys. 22, 1625 (1954).

${ }^{7}$ M. Ichikawa, Acta Crystallogr., Sect. B: Struct. Crystallogr. Cryst. Chem. B34, 2074 (1978).

${ }^{8}$ P. Gilli, V. Bertolasi, V. Ferretti, and G. Gilli, J. Am. Chem. Soc. 116, 909 (1994).

${ }^{9}$ V. Bertolasi, P. Gilli, V. Ferretti, and G. Gilli, Chem.-Eur. J. 2, 925 (1996).

${ }^{10}$ G. Gilli and P. Gilli, J. Mol. Struct. 552, 1 (2000).

${ }^{11}$ J. A. Gerlt and P. G. Gassman, J. Am. Chem. Soc. 115, 11552 (1993).

${ }^{12}$ M. M. Kreevoy and T. M. Liang, J. Am. Chem. Soc. 102, 3315 (1980).

${ }^{13}$ W. W. Cleland, Biochemistry 31, 317 (1992).

${ }^{14}$ W. W. Cleland and M. M. Kreevoy, Science 264, 1887 (1994).

${ }^{15}$ P. A. Frey, S. A. Whitt, and J. B. Tobin, Science 264, 1927 (1994).

${ }^{16}$ P. A. Frey, Magn. Reson. Chem. 39, S190 (2001).

${ }^{17}$ A. Warshel, A. Papazyan, and P. A. Kollman, Science 269, 102 (1995).

${ }^{18}$ S. Scheiner and T. Kar, J. Am. Chem. Soc. 117, 6970 (1995).

${ }^{19}$ A. Novak, Struct. Bonding (Berlin) 18, 177 (1974).

${ }^{20}$ B. Berglund and R. W. Vaughan, J. Chem. Phys. 73, 2037 (1980).

${ }^{21}$ C. A. Klug, P. L. Lee, I.-S. H. Lee, M. M. Kreevoy, R. Yaris, and J. Schaefer, J. Cell. Sci. 101, 8086 (1997).

${ }^{22}$ J. A. Belot, J. Clark, J. A. Cowan, G. S. Harbison, A. I. Kolesnikov, Y.-S. Kye, A. J. Schultz, C. Silvernail, and X. Zhao, J. Phys. Chem. B 108, 6922 (2004).

${ }^{23}$ S. X. Tian and H.-B. Li, J. Phys. Chem. A 111, 4404 (2007).

${ }^{24}$ J. Sørensen, H. F. Clausen, R. D. Poulsen, J. Overgaard, and B. Schiott, J. Phys. Chem. A 111, 345 (2007).

${ }^{25}$ S. F. Darlow and W. Cochram, Acta Crystallogr. 14, 1250 (1961).

${ }^{26}$ S. F. Darlow, Acta Crystallogr. 14, 1257 (1961).

${ }^{27}$ F. Fillaux, N. Leygue, J. Tomkinson, A. Cousson, and W. Paulus, Chem. Phys. 244, 387 (1999).

${ }^{28}$ A. Novak, P. Saumagne, and L. D. C. Bock, J. Chim. Phys. Phys.-Chim. Biol. 60, 1385 (1963).

${ }^{29}$ F. Avbelj, B. Orel, M. Klanjsek, and D. Hadzi, Spectrochim. Acta, Part A 41, 75 (1985).

${ }^{30}$ H. R. Zelsmann, Z. Mielke, and M. M. Ilczyszyn, Spectrochim. Acta, Part A 44, 705 (1988).

${ }^{31}$ M. M. Ilczyszyn, J. Baran, H. Ratajczak, and A. J. Barnes, J. Mol. Struct. 270, 499 (1992).

${ }^{32}$ J. Tomkinson, I. J. Braid, J. Howard, and T. C. Waddington, Chem. Phys. 64, 151 (1982).

${ }^{33}$ J. Howard, J. Tomkinson, J. Eckert, J. A. Goldstone, and A. D. Taylor, J. Chem. Phys. 78, 3150 (1983).

${ }^{34}$ J. Tomkinson, J. Penfold, and J. Howard, J. Mol. Struct. 142, 1 (1986).

${ }^{35}$ N. Kalsbeek, K. Schaumburg, and S. Larsen, J. Mol. Struct. 299, 155 (1993). 
${ }^{36}$ A. Wong, K. J. Pike, R. Jenkins, G. J. Clarkson, T. Anupõld, A. P. Howes, D. H. G. Crout, A. Samoson, R. Dupree, and M. E. Smith, J. Phys. Chem. A 110, 1824 (2006).

${ }^{37}$ M. Fukai, T. Matsuo, and H. Suga, J. Chem. Thermodyn. 20, 1337 (1988).

${ }^{38}$ C. C. Wilson, L. H. Thomas, and C. A. Morrison, Chem. Phys. Lett. 381, 102 (2003).

${ }^{39}$ F. Fillaux, A. Cousson, and J. Tomkinson, Chem. Phys. Lett. 399, 289 (2004).

${ }^{40}$ P. George, C. W. Bock, and M. Trachtman, J. Phys. Chem. 87, 1839 (1983).

${ }^{41}$ M. Garcia-Viloca, A. González-Lafont, and J. M. Lluch, J. Am. Chem. Soc. 119, 1081 (1997).

${ }^{42}$ L. Golič and J. C. Speakman, J. Chem. Soc. 1965, 2530.

${ }^{43}$ D. Hadži, B. Orel, and A. Novak, Spectrochim. Acta, Part A 29, 1745 (1973).

${ }^{44}$ F. Fillaux and J. Tomkinson, Chem. Phys. 158, 113 (1991).

${ }^{45}$ See: http://www-llb.cea.fr

${ }^{46}$ P. W. Betteridge, J. R. Carruthers, R. I. Cooper, C. K. Prout, and D. J. Watkin, J. Appl. Crystallogr. 36, 1487 (2003).

${ }^{47}$ See: http://www.xtl.ox.ac.uk/crystals.html
${ }^{48}$ S. W. Lovesey, Nuclear Scattering, Theory of Neutron Scattered from Condensed Matter (Clarendon Press, Oxford, 1984), Vol. 1.

${ }^{49}$ A. Witkowski, J. Chem. Phys. 47, 3645 (1967).

${ }^{50}$ Y. Maréchal and A. Witkowski, J. Chem. Phys. 48, 3697 (1968).

${ }^{51}$ S. F. Fischer, G. L. Hofacker, and M. A. Ratner, J. Chem. Phys. 52, 1934 (1970).

${ }^{52}$ O. Henri-Rousseau and P. Blaise, The Infrared Spectral Density of Weak Hydrogen Bonds within the Linear Response Theory, Advanced Chemical Physics Vol. 103, edited by I. Prigogine and S. A. Rice (Wiley, New York, 1998), p. 1.

${ }^{53}$ F. Fillaux, A. Cousson, and M. J. Gutmann, J. Phys.: Condens. Matter 18, 3229 (2006).

${ }^{54}$ F. Fillaux, A. Cousson, and M. J. Gutmann, J. Phys.: Condens. Matter 20, 015225 (2008).

${ }^{55}$ M. J. S. Dewar, E. G. Zoebisch, E. F. Healy, and J. J. P. Stewart, J. Am. Chem. Soc. 107, 3902 (1985).

${ }^{56}$ E. Heilbronner, H. Rutishauser, and F. Gerson, Helv. Chim. Acta 42-47, 2285 (1959).

${ }^{57}$ S. Y. Chan and D. Stelman, J. Chem. Phys. 39, 545 (1963).

${ }^{58}$ See: http://www.isis.rl.ac.uk/molecularSpectroscopy/tfxa/ 